
\title{
R Reserach S Suare \\ Concentration Distribution of Anti-VEGF Drugs Injected Into the Vitreous Cavity of Vitrectomy and Non-vitrectomy Eyes
}

\section{Guangsen Liu}

Moorfields Eye Hospital https://orcid.org/0000-0002-6871-6051

\section{Yue Li}

Qindao University Medical College Affiliated Yantai Yuhuangding Hospital

Lei Gao

Weifang Eye Hospital

Xudong Huang ( $\square$ hxd3333@163.com )

Weifang Eye hospital

\section{Research Article}

Keywords: Intravitreal injections, anti-VEGF drugs, pharmacokinetics, vitrectomy

Posted Date: April 27th, 2021

DOI: https://doi.org/10.21203/rs.3.rs-319919/v1

License: (9) This work is licensed under a Creative Commons Attribution 4.0 International License. Read Full License 


\section{Abstract}

Objective: To study the difference of retinal drug concentration distribution of anti-VEGF drugs injected into the vitreous cavity of vitrectomy and non-vitrectomy eyes.

Methods: This article reviews the related studies on the distribution of retinal drugs after vitrectomy and non-vitrectomy eyes after IVI of anti-VEGF drugs.

Results: There were few studies on retinal drug concentration after vitrectomy and non-vitrectomy eyes after vitreous injection of anti-VEGF drugs. Pharmacokinetics plays an important role in drug tissue distribution and onset time. A faster drug clearance rate can theoretically explain that the shorter retention time of anti-VEGF drugs in the vitreous cavity after intraocular injection of anti-VEGF drugs after vitrectomy, the lower concentration of anti-VEGF drugs in the vitreous cavity at the same time point, which can explain the reason for the poor efficacy of anti-VEGF treatment in the eyes after vitrectomy. Therefore, we have reason to believe that there is a difference in retinal drug concentration after vitrectomy and nonvitrectomy eyes after intravitreous injection of anti-VEGF drugs. Previous research results may have some deficiencies, which need to be verified by further research.

Conclusion: There are differences in retinal drug concentration between vitrectomy eyes and nonvitrectomy eyes after vitreous injection of anti-VEGF drugs. There may be some deficiencies in the previous research results, which need to be verified by further research.

\section{Synopsis}

The difference of vitreous cavity fillers will lead to differences in the concentration distribution of drugs injected into the vitreous cavity in the retina.

\section{Introduction}

Anti-vascular endothelial growth factor (anti-VEGF) is a widely used drug for the treatment of intraocular neovascularization in the field of ophthalmology. At present, the commonly used anti-VEGF drugs are pegaptanib sodium (Macugen), bevacizumab (Avastin), ranibizumab (Lucentis), Aflibercept (Eylea), Conbercept (Longmire). Pegaptanib, jointly developed and produced by Eyetech and Pfizer, is the first anti-VEGF drug used in ophthalmology. It was first approved by the Food and Drug Administration (FDA) in 2004 for the treatment of wet age-related macular degeneration (w-AMD)[1]. This is the first time in the world that anti-VEGF drugs have been used in vitreous injection [2]. In 2006, the advent of Ranibizumab became the first treatment in human history to improve the vision of patients with wet-AMD. Subsequently, the number of anti-VEGF by IVI also showed a blowout growth. It is reported that the United States conducted more than 4 million IVI in 2013, and reached an astonishing 5.9 million IVI in 2016.[3] At present, it is not possible to obtain the annual number of IVI in China, but as the most populous country in the world, China has entered the ranks of an aging society and undoubtedly has the largest group of patients receiving IVI of anti-VEGF drugs in the world. 
In addition to the differences in molecular weight and targets of anti-VEGF drugs, the concentration of drugs in retinal lesions may also be one of the important factors affecting the therapeutic effect of patients. Because these drugs are mainly administered through the vitreous cavity and are limited by the special tissue structure of the eye, we have reason to think that the effect of anti-VEGF is mainly due to the direct diffusion and penetration of the drug itself, rather than through the blood transport to the disease site. With the rapid development of vitrectomy, vitrectomy has become the first choice for surgical treatment of complex fundus diseases, and with the increase of the number of vitrectomy operations, the vitreous cavities of many people around us are no longer vitreous but varied although there are a variety of fillers, they will eventually be replaced by water. The vitreous cavity of many patients with fundus diseases is filled with water for life. However, many patients with fundus diseases may have recurrent attacks and need anti-VEGF treatment, but studies have shown that the effect of anti-VEGF treatment after vitrectomy is worse than that without vitrectomy, and there is no sufficient theory to explain why this problem occurs. We assume that it is related to the difference in the anti-VEGF drug concentration distribution of retinas in water and vitreous. However, there is no related research in the past, so it is of great significance for us to choose this study. This paper reviews the retinal concentration distribution of anti-VEGF drugs injected into the vitreous cavity in vitrectomy and non-vitrectomy eyes, hoping to attract the attention of medical workers and lay a foundation for future clinical research.

\section{Previous Research}

Yen-Yi Chen et al treated 148 eyes with diabetic macular edema by intravitreal injection of ranibizumab, of which 46 eyes underwent vitrectomy 3 months ago and 102 eyes did not. The results showed that compared with vitrectomy, non-vitrectomy eyes had the best-corrected visual acuity (BCVA), macular fovea thickness (CFT), and central fovea thickness decreased significantly improved [4]. The results of this study put forward a new direction for us to think about why the effect of anti-VEGF treatment is worse after vitrectomy, and whether it is related to the difference of retinal drug concentration.

There are few studies on the retinal concentration distribution of intravitreal injection of anti-VEGF drugs in vitrectomy eyes and non-vitrectomy eyes. The author found only one related study. The results of SeongJoonAhn et al in 2014 showed that 18 rabbit eyes after vitrectomy were in the experimental group, 18 rabbits without vitrectomy as the control group, and both the experimental group and the control group were given a vitreous injection of Ranibizumab $0.025 \mathrm{ml}(0.25 \mathrm{mg})$. The retinal drug concentration was measured at $1 \mathrm{~h}, 1 \mathrm{~d}, 2 \mathrm{~d}, 5 \mathrm{~d}, 14 \mathrm{~d}$, and $30 \mathrm{~d}$ after injection. The results showed that there was no significant difference in retinal drug concentration between the experimental group and the control group [5].

Similar studies were carried out by Garcia-SalinasR et al to determine whether vitrectomy would affect the distribution of gentamicin sulfate in intravitreous tissues. This study showed that the same type, the same batch, the same amount, and the same concentration of gentamicin sulfate were injected into vitrectomy rabbit eyes (experimental group) and non-vitrectomy rabbit eyes (control group). The concentration of gentamicin sulfate in each tissue was measured at 1, 3, 6, and 12 hours after injection. 
The results showed that the retinal drug concentration in the experimental group was significantly higher than that in the control group 1 hour and 3 hours later. The vitreous cavity drug concentration in the experimental group was significantly lower than that in the control group [6]. However, gentamicin sulfate with high molecular weight is selected in this study, and further studies are needed to confirm whether the results apply to anti-VEGF drugs.

Garcia-SalinasR et al conducted similar studies to determine whether vitrectomy affects the distribution of gentamicin sulfate in vitreous tissues. This study showed that the same type, the same batch, the same amount, and the same concentration of gentamicin sulfate were injected into vitrectomy rabbit eyes (experimental group) and non-vitrectomy rabbit eyes (control group). The concentration of gentamicin sulfate in each tissue was measured at $1,3,6$, and 12 hours after injection. The results showed that the retinal drug concentration in the experimental group was significantly higher than that in the control group after 1 hour and 3 hours. The drug concentration in the vitreous cavity in the experimental group was significantly lower than that in the control group [6]. However, gentamicin sulfate with high molecular weight was selected in this study, and further studies are needed to confirm whether the results apply to anti-VEGF drugs.

\section{Detection Of Retinal Drug Concentration}

How to accurately and effectively determine the tissue distribution of anti-VEGF drugs in the eye is the focus and technical difficulty of the study. Choosing accurate determination methods can improve the accuracy of the research results. At present, there are four main methods for the determination of antiVEGF drugs. Including physical and chemical spectrophotometry, octanoic acid method (BCA method), immunological enzyme-linked immunosorbent assay (ELISA method), and high-performance liquid chromatography (HPLC method) [7]. HPLC method is the most accurate and sensitive method for the determination of anti-VEGF drugs.

The first three methods determine the total protein in the sample, but the monoclonal antibody fermentation broth often contains a lot of pigment and host protein, the application of these three methods can not accurately determine the antibody content in the fermentation broth, so these methods have some limitations. At present, the expression of monoclonal antibody in the fermentation broth is above gram level [8], while the concentration of ELISA method is less than microgram level, so the detection drug needs to be diluted to a high multiple, so the accuracy of ELISA method is reduced.

Chromatography was first discovered in 1906 when Russian botanist Tswett studied the separation of plant pigments with calcium carbonate, hence the name chromatography. HPLC method is a new detection method that came out in 1960. This method can realize the separation and determination of samples with high speed, high efficiency, and high sensitivity. HPLC uses liquid as a mobile phase and adopts a high-pressure infusion system to pump single solvent with different polarity or different proportions of mixed solvent and buffer into the chromatographic column equipped with fixed phase. After each component in the column is separated, it goes to the detector for detection, to realize the 
analysis of the sample. HPLC has the characteristics of high speed, high efficiency, high sensitivity, can be reused, a small number of samples, easy to recover, and so on. Since the HPLC method was included in the Chinese Pharmacopoeia in 1985, the application of the HPLC method in drug inspection has increased rapidly, and its role in drug quality control has become increasingly obvious [9].

\section{Discussion}

According to the results of the National Bureau of Statistics, the total population of China in 2017 was 1.4 billion. In terms of age composition, China has 240.9 million people aged 60 and above, accounting for $17.3 \%$ of the total population, of which 158.31 million are aged 65 and above, accounting for $11.4 \%$ of the total population. The number of elderly people in China accounts for about $20 \%$ of the world's total elderly population, according to international standards[10]. The proportion of people over 60 years old in a country or region is more than $10 \%$, and the proportion of people over 65 years old is more than $7 \%$, which marks the entry of an aging society. AMD is the most common cause of blindness in people aged 55 and above, of which 55\%-88\% of AMD patients develop "legal blindness" (best-corrected visual acuity $<20$ amp 200) after the age of 70[11]. With the progress of the aging population, more and more AMD patients need to be treated in our country. If AMD can not be treated in time and eventually become blind, it will have an indelible impact on the quality of life and mental health of elderly patients, and bring a heavy burden to the family and society.

The advent of anti-VEGF has brought great good news for AMD patients. From the initial control of the disease to the later development of ranibizumab, it can improve the vision of AMD patients. Anti-VEGF drugs are more and more widely used in clinical ophthalmology, but the clinical efficacy of anti-VEGF drugs varies greatly. With the emergence of vitrectomy, This surgical method is recognized by more and more ophthalmologists because of its minimally invasive and efficient advantages, and its clinical application is becoming more and more common. More and more patients after vitrectomy are faced within our work, and most of them are elderly patients. This group is more likely to receive anti-VEGF therapy after surgery. Previous studies have shown that the effect of anti-VEGF treatment after vitrectomy is worse than that of non-vitrectomy, but few studies can explain why vitreous injection of anti-VEGF drugs is less effective after vitrectomy. This is a practical problem to be solved in the future. Finding the root cause of the problem will help to improve the effect of anti-VEGF therapy after vitrectomy.

Concerning the effect of anti-VEGF drug treatment, the first thing we should think of is the temporal and spatial distribution of anti-VEGF drugs in the eyes. The higher concentration of the drug reaches the focus and the longer it takes to act on the focus, the more reason we have to believe that the treatment is more effective, and the only study we found shows that intravitreal injection of ranibizumab is given to rabbit eyes after vitrectomy and non-vitrectomy eyes. There was no significant difference in retinal drug concentration at different time points [12]. This study showed that there was no difference in the temporal and spatial distribution of intravitreal injection of ranibizumab between vitrectomy and nonvitrectomy eyes. However, there are the following problems in this study: 1 . The sample size of the study is small, and the results are poorly representative, which needs to be verified by further large sample 
studies. 2. In this study, only one anti-VEGF drug, ranibizumab, can not be used to explain the distribution of all anti-VEGF drugs in the retina, because different anti-VEGF drugs have different molecular weight, physical and chemical properties, metabolic effects, and so on. 3. A variety of anti-VEGF drugs should be selected for further study. The study was carried out in the rabbit eye in vivo, and finally, the rabbit was killed and the retinal tissue was taken to determine the concentration of ranibizumab. Although the anatomical structure of the rabbit eye is similar to that of the human eye, it is not a human eye. Whether the conclusion drawn in the animal eye experiment applies to the human eye remains to be studied, but limited to the limitations of the existing anti-VEGF drug concentration detection methods, there is no method to detect the retinal concentration of anti-VEGF drugs in vivo. It is contrary to medical ethics, so it is not suitable for human eye research. At present, the detection method of intraocular concentration of anti-VEGF drugs should be further studied to realize the detection of retinal drug concentration in vivo.

Pharmacokinetics plays an important role in drug tissue distribution and onset time. Studies have shown that the drug clearance rates of bevacizumab, ranibizumab, triamcinolone acetonide, ciprofloxacin, amikacin, ceftazidime, vancomycin, and amphotericin B in eyes after vitrectomy are higher than those in non-vitrectomy eyes[13-19]. A faster drug clearance rate can theoretically explain that the shorter retention time of anti-VEGF drugs in the vitreous cavity after intraocular injection of anti-VEGF drugs after vitrectomy, the lower concentration of anti-VEGF drugs in the vitreous cavity at the same time point, which can explain the reason for the poor efficacy of anti-VEGF treatment in the eyes after vitrectomy. Therefore, we have reason to believe that there is a difference in retinal drug concentration after vitrectomy and nonvitrectomy eyes after intravitreous injection of anti-VEGF drugs. Previous research results may have some deficiencies, which need to be verified by further research.

To sum up, at present, anti-VEGF drugs also play an important role in the eyes after vitrectomy, but the research on the distribution of drugs in the retina after vitrectomy and non-vitrectomy eyes is blank. Revealing the difference of retinal drug concentration between the two eyes is helpful to guide the treatment of anti-VEGF after vitrectomy. It has important guiding significance to obtain better therapeutic effects for more and more patients who need anti-VEGF therapy after vitrectomy.

\section{Declarations}

Acknowledgments

Funding This study has not any financial support.

Compliance with ethical standards.

Conflict of interest They authors declare that they have no conflict of interest.

Ethical approval All procedures performed in studies involving human participants were in accordance with the ethical standards of the institutional ethical committee and with the 1964 Helsinki Declaration and its later amendments or comparable ethical standards. 
Informed consent Informed consent was obtained from all individual participants included in the study.

\section{References}

1. Brown DM, Kaiser PK, Michels M, Soubrane G, Heier JS, Kim RY, Sy JP, Schneider S; ANCHOR Study Group. Ranibizumab versus verteporfin for neovascular age-related macular degeneration. N Engl J Med. 2006 Oct 5;355(14):1432-44. doi: 10.1056/NEJMoa062655. PMID: 17021319.

2. Eyetech Study Group. Anti-vascular endothelial growth factor therapy for subfoveal choroidal neovascularization secondary to age-related macular degeneration: phase II study results. Ophthalmology. 2003 May;110(5):979-86. doi: 10.1016/S0161-6420(03)00085-X. PMID: 12750101.

3. Suskind AM, Clemens JQ. Health policy 2016: implications for geriatric urology. Curr Opin Urol. 2016 Mar;26(2):207-12. doi: 10.1097/MOU.0000000000000264. PMID: 26765043; PMCID: PMC4780417.

4. Chen YY, Chen PY, Chen FT, Chen YJ, Wang JK. Comparison of efficacy of intravitreal ranibizumab between non-vitrectomized and vitrectomized eyes with diabetic macular edema. Int Ophthalmol. 2018 Feb;38(1):293-299. doi: 10.1007/s10792-017-0462-1. Epub 2017 Feb 7. PMID: 28176171.

5. Ahn SJ, Ahn J, Park S, Kim H, Hwang DJ, Park JH, Park JY, Chung JY, Park KH, Woo SJ. Intraocular pharmacokinetics of ranibizumab in vitrectomized versus nonvitrectomized eyes. Invest Ophthalmol Vis Sci. 2014 Jan 29;55(1):567-73. doi: 10.1167/iovs.13-13054. Erratum in: Invest Ophthalmol Vis Sci. 2015 Mar;56(3):1473-4. Dosage error in article text. PMID: 24398088.

6. Garcia-Salinas R, Hasany S, Chisholm L, Basu PK, Moffat K, Harvey P. Effect of vitrectomy on the ocular distribution of gentamicin in the rabbit. Can J Ophthalmol. 1991 Jun;26(4):196-9. PMID: 1889020.

7. Zhang T Y, Quan C , Dong M W . HPLC for Characterization and Quality Control of Therapeutic Monoclonal Antibodies[J]. LC GC Europe, 2014, 32(11):796-808.

8. Humphreys DP, Glover DJ. Therapeutic antibody production technologies: molecules, applications, expression and purification. Curr Opin Drug Discov Devel. 2001 Mar;4(2):172-85. PMID: 11378956.

9. Lima E S , Abdalla D S P. High-performance liquid chromatography of fatty acids in biological samples - ScienceDirect[J]. Analytica Chimica Acta, 2002, 465(1-2):81-91.

10. Jiandong, Chen, Yun, et al. The influences of aging population and economic growth on Chinese rural poverty[J]. Journal of Rural Studies, 2016.

11. Sunness JS, Massof RW, Johnson MA, Bressler NM, Bressler SB, Fine SL. Diminished foveal sensitivity may predict the development of advanced age-related macular degeneration. Ophthalmology. 1989 Mar;96(3):375-81. doi: 10.1016/s0161-6420(89)32883-1. PMID: 2710529.

12. Ahn SJ, Ahn J, Park S, Kim H, Hwang DJ, Park JH, Park JY, Chung JY, Park KH, Woo SJ. Intraocular pharmacokinetics of ranibizumab in vitrectomized versus nonvitrectomized eyes. Invest Ophthalmol Vis Sci. 2014 Jan 29;55(1):567-73. doi: 10.1167/iovs.13-13054. Erratum in: Invest Ophthalmol Vis Sci. 2015 Mar;56(3):1473-4. Dosage error in article text. PMID: 24398088. 
13. Funatsu H, Yamashita H, Sakata K, Noma H, Mimura T, Suzuki M, Eguchi S, Hori S. Vitreous levels of vascular endothelial growth factor and intercellular adhesion molecule 1 are related to diabetic macular edema. Ophthalmology. 2005 May;112(5):806-16. doi: 10.1016/j.ophtha.2004.11.045. PMID: 15878060.

14. Brown DM, Nguyen QD, Marcus DM, Boyer DS, Patel S, Feiner L, Schlottmann PG, Rundle AC, Zhang J, Rubio RG, Adamis AP, Ehrlich JS, Hopkins JJ; RIDE and RISE Research Group. Long-term outcomes of ranibizumab therapy for diabetic macular edema: the 36-month results from two phase III trials: RISE and RIDE. Ophthalmology. 2013 Oct;120(10):2013-22. doi: 10.1016/j.ophtha.2013.02.034. Epub 2013 May 22. PMID: 23706949.

15. Schmidt-Erfurth U, Lang GE, Holz FG, Schlingemann RO, Lanzetta P, Massin P, Gerstner O, Bouazza AS, Shen H, Osborne A, Mitchell P; RESTORE Extension Study Group. Three-year outcomes of individualized ranibizumab treatment in patients with diabetic macular edema: the RESTORE extension study. Ophthalmology. 2014 May;121(5):1045-53. doi: 10.1016/j.ophtha.2013.11.041. Epub 2014 Feb 1. PMID: 24491642.

16. Bressler SB, Glassman AR, Almukhtar T, Bressler NM, Ferris FL, Googe JM Jr, Gupta SK, Jampol LM, Melia M, Wells JA 3rd; Diabetic Retinopathy Clinical Research Network. Five-Year Outcomes of Ranibizumab With Prompt or Deferred Laser Versus Laser or Triamcinolone Plus Deferred Ranibizumab for Diabetic Macular Edema. Am J Ophthalmol. 2016 Apr;164:57-68. doi: 10.1016/j.ajo.2015.12.025. Epub 2016 Jan 21. PMID: 26802783; PMCID: PMC4811755.

17. Rajendram R, Fraser-Bell S, Kaines A, Michaelides M, Hamilton RD, Esposti SD, Peto T, Egan C, Bunce C, Leslie RD, Hykin PG. A 2-year prospective randomized controlled trial of intravitreal bevacizumab or laser therapy (BOLT) in the management of diabetic macular edema: 24-month data: report 3. Arch Ophthalmol. 2012 Aug;130(8):972-9. doi: 10.1001/archophthalmol.2012.393. PMID: 22491395.

18. Kakinoki M, Sawada O, Sawada T, Saishin Y, Kawamura H, Ohji M. Effect of vitrectomy on aqueous VEGF concentration and pharmacokinetics of bevacizumab in macaque monkeys. Invest Ophthalmol Vis Sci. 2012 Aug 24;53(9):5877-80. doi: 10.1167/iovs.12-10164. PMID: 22836776.

19. Christoforidis JB, Williams MM, Wang J, Jiang A, Pratt C, Abdel-Rasoul M, Hinkle GH, Knopp MV. Anatomic and pharmacokinetic properties of intravitreal bevacizumab and ranibizumab after vitrectomy and lensectomy. Retina. 2013 May;33(5):946-52. doi: 10.1097/IAE.0b013e3182753b12. PMID: 23407351; PMCID: PMC4086838. 